\title{
The effect of encoding training on perceptual recall'
}

\author{
MURRAY GLANZER, NEW YORK UNIVERSITY \\ JOSEPH FLEISHMAN, YESHIVA UNIVERSITY
}

The effect of encoding training on tachistoscopic performance was measured by training groups of $S s$ in either an octal or a standardized English encoding of nine place binary numbers. A control group of $S$ s was allowed to encode ad lib during an equivalent training period. Performance was measured before, during and after the encoding training. It was found that imposing a code on $S$ impaired tachistoscopic performance both during and for some time after the encoding training. Possible reasons for this effect are discussed.

The purpose of this experiment was to determine the effects of encoding training on performance in a perceptual task. A recent proposal (Glanzer \& Clark, 1963a, b, 1964) has emphasized the effect of the S's code on the efficiency with which $S$ handles perceptual information. This proposal, the verbal loop hypothesis, asserts that the $s$ faced with a perceptual task such as recall of a visual display encodes the information verbally and then translates the verbal information into a final response. According to this hypothesis, the form of the code and, in particular, the length of the code for individual items plays a critical role. In support of the proposal, evidence has been presented for the predicted relathons between verbal code length and measures of perceptual difficulty and organization.

The verbal loop hypothesis is closely related to an earlier proposal made by Miller (1956) that recoding or "chunking" facilitates memory. In support of the proposal Miller cited data from an immediate recall experiment by Sidney Smith. Further support was furnlshed by Pollack and Johnson (1965) in an experiment similar to Smith's, on the recall of auditorlly presented sequences of binary numbers. Two attempts to demonstrate similar effects in tachistoscopic performance, 1.e., responses to fast, simultaneous, visual presentations, have falled (Klemmer, 1564; Pollack \& Johnson, 1965). Both experiments, however, present certain difficulties. In the Pollack and Johnson experiment, the Ss were not really constrained to make use of the prescribed coding until they had completed their response. In the Klemmer experiment, a complex sequence of conditions was used, starting with binary cued response and followed by alternating cued and uncued (full) octal responses to the eight patterns. It is not completely clear, from the data, that there is no advantage of octal cued over binary cued or octal uncued over binary uncued.

The present study aimed to get a fuller picture of the effects of encoding training on tachistoscopic performance. The fuller picture was, in part, obtained by including a control group that was not present in the preceding studies.

The verbal loop hypothesis suggests the following possibilities: (1) that verbal training should change S's efficiency in perceptual tasks; (2) more spectfically, that training in efficient codes, codes that minimize the number of words that encode each input, should improve perceptual performance. To evaluate these possibilities, the performance of three groups that varied in their encoding training was compared.

\section{Method}

There were three stages in the experiment. During the first stage Ss' ability to reproduce briefly exposed 9-digit binary numbers was measured. Binary numbers were used because they are easy to present and record and show the same systematic, perceptual organization effects as other classes of stimuli. They range from simple, well-organized sequences, e.g., 111111111, to complex, poorly-organized sequences, e.g., 110100101. During the second stage the Ss were trained on one of three types of coding. During the third stage, the effect of the encoding training was measured by again measuring the $\mathrm{Ss}^{\prime}$ ability to reproduce the numbers.

\section{Subjects}

The Ss were 11 college students who were paid for their participation.

\section{Apparatus and Waterials}

. The stimulus material was presented on a $5 \times 5$ ft screen with a Keystone slide projector. The duration of the slide exposure was determined by a Wollensak shutter. The main stimuli were the 512 9-place binary numbers from 000000000 to 111111111 . There were also stimuli used for practice and training that consisted of the eight 3-place numbers from 000 to 111. All binary numbers were on $35 \mathrm{~mm}$ slides, printed black against a blue background.

\section{Procedure}

The Ss were trained Individually and tested in small groups of two to five, as indicated below. Training and testing were carried out in a semtdarkened room. There were three main stages in the experiment-preliminary or pretraining tests, training, and final or posttraining tests. 
Pretraining tests, five days. The purpose of the pretraining was to establish and measure stable performance by the $\mathrm{Ss}$ on the perceptual task and to afford a basis for matching groups of Ss. The Ss were run in small groups. During the first day stimuli were presented to Ss in the following order: four randomized practice sets of 3-digit binary numbers (eight numbers per set) followed by the main series of 512 randomized 9-digit binary numbers. The Ss were instructed to reproduce each number immediately in booklets that contained 32 blanks per page, each blank being identified by a letter. Each binary number was preceded by the $E^{\prime}$ 's stating the identifying letter. The order and timing of events was as follows: (1) E calling out the identifying letter -about $0.5 \mathrm{sec}$; (2) dark period (slide change) -2.5 sec; (3) binary number $-0.5 \mathrm{sec}$ (4) answer period -approximately $20 \mathrm{sec}$. On the four subsequent days, the procedure was the same except that the 3-digit practice sets were omitted. The 9-digit binary numbers were presented in a different randomized order on each day. No oral responses were permitted. No feedback was given as to the correctness of individual responses.

At the end of the fifth pretraining day, the Ss were assigned to one of the three experimental groups on the basis of their scores on the fourth and fifth sessions. The assignment was designed to give three groups matched on mean performance.

Training period, nine days. The purpose of the training was to impose different types of encoding on the Ss. The Ss were individually trained to describe the numbers orally in one of three codes -Control, English-Fixed, or Octal. The Control group, four Ss, was permitted to use their own individual description of the numbers. They were informed that any description was acceptable as long as it was accurate. The English-Fixed group, three Ss, was trained to describe the numbers in a standard form consisting of three phrases. Each phrase described three digits, grouped into runs. Examples of the English-Fixed verbalizations are the following: for 110100000-"two ones, an oh; a one, two ohs; three ohs;" for 011100111-"an oh, two ones; a one, two ohs; three ones." The Octal group, four Ss, was trained to translate each number into three octal numbers. They would encode the two binary numbers given above as "six, four, oh" and "three, four, seven." The procedure was subject-paced throughout for all three groups, with the S pressing a projector control button to determine when each stimulus number appeared. $E$ gave $S$ feedback as to the correctness of each response. When $S$ made an error, $E$ read off the correct response in the form that $\mathrm{S}$ was using for his responses. In the control condition, E would use whatever general form of verbalization $\mathbf{S}$ had adopted.
During the first day, each $\mathrm{S}$ was given preliminary training with 384 3-digit binary numbers. There were 32 presentations at $0.5 \mathrm{sec}$ duration, then 224 at .20 $\mathrm{sec}$ duration and 128 at $.10 \mathrm{sec}$ duration. During the second day, 64 3-place numbers were presented at $.10 \mathrm{sec}$ exposure for further encoding training; these were then followed by four 9-digit practice numbers and then 256 9-digit numbers, half of a complete randomization, all at $.50 \mathrm{sec}$ exposure. Days 3 to 7 were run in the same fashion with 256 numbers shown, but 3-digit numbers were no longer presented. On Days 8 and 9 all 512 9-place binary numbers in a randomization were shown in each session. (By this time Ss' performance was fast enough to permit the presentation of a larger number of stimuli.) During the training, all the 512 9-place binary numbers were shown five times.

Postraining procedure, five days. The procedure was identical with that of the pretraining procedure. The Ss were run in small groups, at $0.5 \mathrm{sec}$ exposure, with the only response required being the reproduction of the numbers in answer booklets. As in the pretraining, no oral responses were permitted. No feedback was given. In each session, a different randomization of the 512 numbers was presented. The presentation rate was controlled by $\mathrm{E}$.

\section{Results}

Each response was scored as either correct or incorrect, with no part scores. The curves for the group means are plotted in Fig. 1. As can be seen, the groups, since they were matched, are very similar in their mean performance during the pretraining. With the onset of the training, the curves of the groups diverge. The Octal group's errors increase markedly. The English-Fixed group's errors also increases, but less markedly. The Control group's curve seems to be a simple continuation of its course during pretraining. The differences that appear in the first training block continue through the succeeding training blocks with the Octal group showing the greatest number of errors and the Control group showing the fewest on each of the training blocks. These differences between the groups are maintained when they are tested during the posttraining period, when all Ss gave the same type of written response. The Octal group makes more errors than either the English-Fixed or the Control group. The English-Fixed group makes more errors than the Control on the first two posttraining test days. Thereafter, the means for these two groups are reversed.

Within each group, at each stage of the experiment, there were very marked individual differences in performance. For example, on the fifth day of pretraining, the total number of errors per $S$ ranged from 50 to 360 . These differences are highly reliable. 




Fig. 1. Mean number of errors on the successive blocks of trials by the Octal (O), English-Fixed (E), and Control (C) groups.

The correlations between the Ss' scores on different days range between .829 and .966 with a mean of .911 .

Account was taken of these large, reliable individual differences by using analysis of covariance to analyze the differences between groups during the training and posttraining periods. The control variable used was the sum of errors for each individual over the five pretraining sessions. With the analysis of covariance, the difference between the three groups during training is statistically significant $(F=7.875$, $\mathrm{df}=2 / 7, \mathrm{p}<.025)$.

The interpretation of performance during training is, however, complicated by the fact that different final responses were made by the $\mathrm{Ss}$ in different groups. This complication is eliminated in the posttraining testing, since the Ss make the same type of final response-writing the number as digits. Analysis of covariance of the difference between the means for the three groups, using the sum of scores over the five posttraining blocks, gives an $F=4.703(d f=2 / 7, .05<p<.10)$. The sum of scores over the five blocks includes, however, several days of near asymptotic performance by two of the groups. If the blocks of posttraining trials are tested separately, the results are as follows ( $\mathrm{d} f=2 / 7$ in all cases): Block 1, $F=5.123$ ( $p<.05)$; Block 2, $F=39.856$ $(p<.005) ;$ Block 3, F=4.96 ( $p<.05) ;$ Block 4, $F=3.06$ (ns); Block 5, F $<1$.

There is evidence of a perturbation in performance in the shifts from training to posttraining. Ten of the 11 Ss show an increase in errors from the last trial of the training block to the first trial of the posttraining block. A simple binomial test of the probability of 10 increases out of 11 instances with binomial $p=q=.50$, gives a significant effect $(p<.05)$.

The Control Ss' verbalizations showed change, the lengths of the verbalizations declining over the five trial blocks. The mean verbalization lengths for the five training blocks were as follows: $8.43,8.61,8.52$, 8.12, 7.96.

\section{Discussion}

The results give support to only the first possibility listed earlier. Encoding training does have a clear effect on the S's performance. This effect continues even when the $S$ is no longer required to give the overt response involved in the encoding training. The second possibility was not supported. Training in economical or short codes does not give superior performance. Indeed, the octal code, which is the shortest, gives the poorest performance both during training and in the posttraining testing.

The mean code lengths of the prescribed codes for the experimental Ss during training are as follows: Octal-3 words; English-fixed-12 words. The mean ad lib verbalization length for the Control Ss was 8.20.

The difficulty with the Octal code cannot be ascribed solely to the grouping by threes that it imposed on S. Grouping by threes was also imposed by the English-Fixed code. This code which has the longest average code length gives consistently better performance than the Octal code, performance that approaches that of the Control group.

The finding that there is no facilitative effect of octal encoding on tachistoscopic performance agrees with the findings of Klemmer (1964) and Pollack and Johnson (1965). The finding that there is actually a detrimental effect of such encoding has not, however, been demonstrated in earlier studies.

Klemmer used patterns formed by a line of 21 lights arranged in seven groups of three each. The Ss reported these in binary form-" 'one' for on and 
'oh' for off'" (p. 19)-or in octal form. Both were imposed forms of coding. Klemmer found little or no effect of extensive training on the octal code. In the light of the present study, it can be asserted that if Klemmer had also collected data for Ss giving ad lib reports he would have obtained performance considerably better than under binary or octal report. The binary report form in which each light is reported separately probably hampers the $S$ as much as the octal report form.

Pollack and Johnson (1965) found effects of encoding training on (auditory) memory tasks but not on tachistoscopic tasks. The data they present do not show the adverse effects of encoding training on tachistoscopic performance found in the present study. Their Ss, however, could avold the direct use of the imposed code, recording their responses before they gave the prescribed form (Pollack \& Johnson, p. 610).

The results suggest that forcing $s$ to replace his usual code with a new code has not been accomplished, even with rather lengthy training. It seems likely that what the Ss in the Octal and English-Fixed groups did was to add another encoding step in processing the information. This additional step can facilitate performance when there is ample time as in the serially presented auditory memory tasks used by Pollack and Johnson and by Smith. In fast, visual tasks, however, the extra step reduces S's efficiency. There are several possible reasons for this effect. One is that the limited time does not permit $S$ to complete his encoding. Another is that the extra step makes possible additional errors. A third is, assuming there are two successive coding steps, that the two coding systems interfere with each other.

It may be possible that more extensive training would give a replacement of the S's habitual coding procedure with a new code. The fact that the groups seem to be approaching different asymptotes argues against this. However, the experimental Ss were receiving training during a relatively short period each day and spending the rest of the day in an environment in which the experimental codes were not being used.

In summary, imposing any code has a clear negative effect on tachistoscopic performance. This holds during training and carries over into subsequent performance. It was argued that the imposed codes did not replace the S's preferred or customary code but added an additional step in the information processing. The effect on tachistoscopic performance is markedly different from that found with sequentially presented binary numbers that involve a relatively long storage of the input information. There $S$ may have sufficient time to complete the encoding and may need the economical form that the Octal code supplies. Whether a training procedure can be devised or can be carried out for a sufficient length of time to replace the S's customary encoding form remains to be seen.

\section{References}

Glanzer, M., \& Clark, W. H. Accuracy of perceptual recall: An analysis of organization. J. verbal Learn. verbal Behav., 1963a, 1, 289-299.

Glanzer, M., \& Clark, W. H. The verbal loop hypothesis: Binary numbers. $J$. verbal Learn. verbal Behav., 1963b, 2, 301-309.

Glanzer, M., \& Clark, W. H. The verbal loop hypothesis: Conventional figures. Amer. J. Psychol., 1964, 77, 621-626.

Klemmer, E. T. Does recoding from binary to octal improve the perception of binary patterns? J. exp. Psychol., 1964, 67, 19-21.

Miller, G. A. The magical number seven, plus or minus two: Some limits on our capacity for processing information. Psychol. Rev., 1956, 63, 81-97.

Pollack, I., Johnson, L. B. Memory-span with efficient coding procedures. Amer. J. Psychol., 1965, 78, 609-614.

\section{Note}

1. This investigation was supported by the U. S. Army Medical Research and Development Command, Department of the Army, under Research Contract DA-49-193 MD-2496.

(Accepted for publication August 3, 1967.) 